OPEN ACCESS

Edited by:

Kuzhuvelil B. Harikumar,

Rajiv Gandhi Centre for

Biotechnology, India

Reviewed by:

Dawn Sijin Nin,

National University of

Singapore, Singapore

Guruvayoorappan Chandrasekharan,

Regional Cancer Center

Thiruvananthapuram, India

*Correspondence:

Songlin Zhou

zhousonglin106@163.com

${ }^{\dagger}$ These authors have contributed equally to this work

Specialty section:

This article was submitted to

Cancer Molecular Targets and

Therapeutics,

a section of the journal

Frontiers in Oncology

Received: 30 September 2019

Accepted: 22 January 2020

Published: 11 February 2020

Citation:

Wang M, Zhao H, Hu J, Xu Z, Lin Y and Zhou S (2020) Penicilazaphilone

C, a New Azaphilone, Induces

Apoptosis in Gastric Cancer by

Blocking the Notch Signaling Pathway.

Front. Oncol. 10:116

doi: 10.3389/fonc.2020.00116

\section{Penicilazaphilone C, a New} Azaphilone, Induces Apoptosis in Gastric Cancer by Blocking the Notch Signaling Pathway

\author{
Ming Wang ${ }^{1,2 \dagger}$, Huange Zhao ${ }^{1 \dagger}$, Juanjuan $\mathrm{Hu}^{3}$, Zhen $\mathrm{Xu}{ }^{1}$, Yingying $\mathrm{Lin}^{1}$ and Songlin Zhou ${ }^{1 *}$ \\ ${ }^{1}$ Key Laboratory of Tropical Translational Medicine of the Ministry of Education \& Hainan Provincial Key Laboratory of Tropical \\ Medicine, Hainan Medical University, Haikou, China, ${ }^{2}$ Health and Family Planning Commission of Wanzai County of Jiangxi \\ Province, Yichun, China, ${ }^{3}$ Department of Medical Insurance Service, Third Affiliated Hospital of Nanchang University, \\ Nanchang, China
}

Penicilazaphilone $\mathrm{C}(\mathrm{PAC})$ is a novel azaphilonidal derivative isolated by our group that demonstrates good anticancer activities. Considering that its molecular mechanisms remain largely unknown, here we explore the molecular mechanisms of the anticancer activities of PAC against gastric cancer. The in vitro effects of PAC on cell growth, proliferation, and apoptosis were evaluated by MTT, BrdU, MTS, colony formation assays, Hoechst 33258 staining, and flow cytometry. Related proteins were examined by western blotting. Notch receptor expression was analyzed by RT-PCR. In vivo antitumor activities of PAC were observed in a nude mouse model. We found that compared to the controls, PAC treatment suppressed cell proliferation and promoted apoptosis in MGC-803 and SGC-7901 cells, and the Notch/PTEN/AKT axis was involved in the activating PAC-induced apoptosis. PAC treatment led to decreased levels of Notch (NTM), NICD, PPTEN, and pAKT compared to controls. PAC-induced inhibition of Notch-related protein expression levels and the resulting apoptosis were reversed by overexpression of Notch1 (NTM) or/and Notch2 (NTM). Moreover, PAC treatment clearly inhibited tumor growth in mice both bearing tumors derived from both MGC-803 and SGC-7901 cells. This work reveals that PAC induces the apoptosis by suppressing activation of Notch receptor proteolytic cleavage and subsequently blocking the PTEN/AKT signaling axis in gastric cancer cells. Thus, PAC is a potential alternative agent for the treatment of gastric cancer.

Keywords: penicilazaphilone C, notch signaling pathway, gastric cancer, apoptosis, molecular mechanism

\section{INTRODUCTION}

Gastric cancer (GC) is a very common malignant tumor that is ranked the fourth leading cause of cancer death globally (1), and the morbidity and mortality of GC rank second in China, according to the National Cancer Institute of China $(2,3)$. Although new anticancer drugs and therapeutic strategies are being increasingly used for GC therapy, overall survival rates of GC are still unsatisfactory, with high incidences and deaths among GC patients, which have not decreased as anticipated $(4,5)$. Therefore, exploring more effective therapeutic strategies or new anticancer drugs is urgently required to treat this cancer or improve the quality of life of GC patients. 
Penicilazaphilone C (PAC, Figure 1A) is a novel natural product that was isolated by our research group from the marine fungus Penicillium sclerotiorum M-22 (6). PAC is a novel azaphilone with a special pyranoquinone bicyclic core structure (6). Previous studies have shown that azaphilone molecules exert a variety of important biological activities (7-9). Indeed, PAC

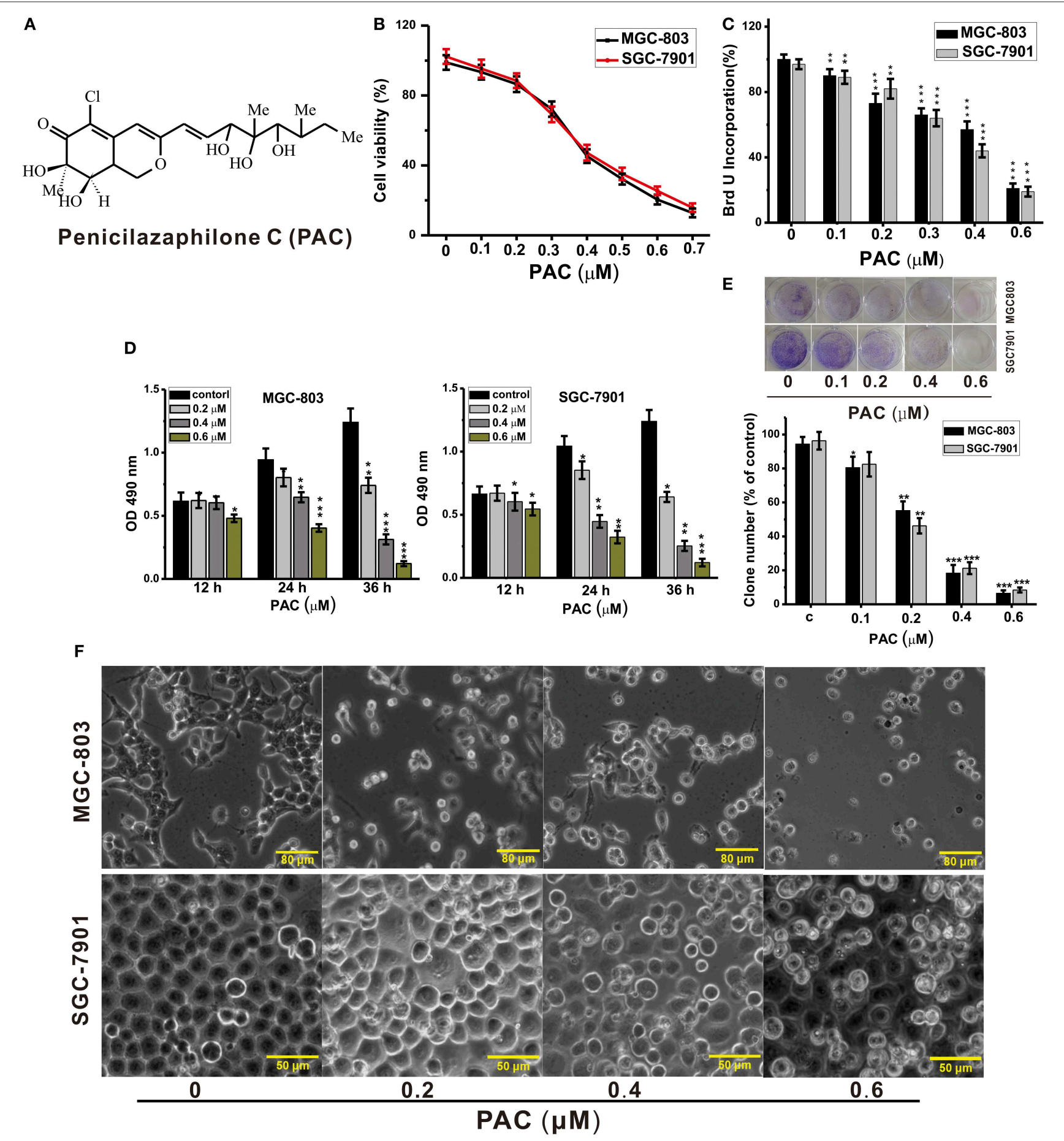

FIGURE 1 | PAC inhibits the proliferation of gastric cancer cells. (A) Structure of penicilazaphilone C (PAC). (B) MGC-803 and SGC-7901 cells were treated with the indicated concentrations of PAC for $24 \mathrm{~h}$, and cell viability was measured with an MTT assay. (C) Cells were treated for $24 \mathrm{~h}$ as in (B), and disrupted cell membranes were detected with a BrdU assay. (D) Cells were treated as in (B) for 12, 24, and $36 \mathrm{~h}$, and MTS-incorporating live cells were detected with an MTS proliferation assay. (E) Cells were treated as in (B) for 14 days, and live cells were detected with a colony formation assay. (F) Cells were treated as in (B) for $24 \mathrm{~h}$; cell morphology was observed under an inverted phase-contrast microscope and images were obtained. Data are expressed as the mean \pm SD; ${ }^{\star} P<0.05,{ }^{* *} P<0.01,{ }^{* \star *} P<0.001$. 
showed highly selective cytotoxicity in GC cells in our previous study (6). However, the molecular mechanism by which PAC inhibits GC cells remains unclear.

The Notch signaling pathway is widely distributed among vertebrates and invertebrates and is highly evolutionarily conserved (10). Notch signaling affects multiple normal morphologenic processes, including the differentiation of pluripotent progenitor cells, apoptosis, cell proliferation, and cell boundary formation $(11,12)$. Humans and rodents have four Notch receptors (Notch 1-4) and five ligands (Jagged 1, 2, Delta-like 1, 3, 4,) (13). The Notch receptor is composed of an extracellular region, a single transmembrane region, and an intracellular region containing an ankyrin domain and a RBP-JK-associated molecule domain (14). Notch signaling is activated by interaction of a ligand from adjacent cells with the Notch receptor (14). Two cleavage events release the intracellular domain of the Notch receptor (NICD) into the cytoplasm, after which it enters the nucleus and binds to the transcription factor CSL to activate the NICD/CSL transcription complex, thereby inducing expression of target genes, such as HES, HEY, and HERP, and exerting biological effects (13). In this study, we investigated the therapeutic effect of PAC on GC in detail to identify the underlying mechanism of PAC-mediated inhibition of GC cell proliferation.

\section{MATERIALS AND METHODS}

\section{Cell Lines and Culture}

The human GC cell lines MGC-803, HGC-27, AGS, MKN45, and SGC-7901 and the normal gastric mucosa cell line GES-1 were obtained from ATCC (Manassas, USA). The cells were cultured in DMEM or RPMI-1640 (HyClone, USA), supplemented with $10 \%$ fetal bovine serum, $100 \mu \mathrm{g} / \mathrm{mL}$ streptomycin, and $100 \mathrm{U} / \mathrm{mL}$ penicillin (HyClone, USA) at $37^{\circ} \mathrm{C}$ in a humidified incubator containing $5 \% \mathrm{CO}_{2}$. Cells were passaged at $80-90 \%$ confluence and were used for experiments in the exponential growth phase.

\section{Cell Viability and Proliferation Assays}

Cells were incubated in 96-well plates and exposed to the indicated concentration of PAC $(0.1,0.2,0.3,0.4,0.5,0.6,0.7 \mu \mathrm{M})$ or vehicle control (DMSO) for $24 \mathrm{~h}$. Cell viabilities were evaluated with an MTT assay (Sigma, USA). In brief, $10 \mu \mathrm{L}$ of $5 \mathrm{mg} / \mathrm{mL}$ MTT was added to each well and incubated for $4 \mathrm{~h}$. The medium was replaced with $150 \mu \mathrm{L}$ of DMSO to dissolve the crystal formazan dye and absorbance was detected at $540 \mathrm{~nm}$ using an ELX808IU Microplate Reader (BioTek, USA).

Cells were seeded in 96-well plates and exposed to the indicated concentration of PAC $(0.1,0.2,0.3,0.4,0.6 \mu \mathrm{M})$ or vehicle control (DMSO) for $24 \mathrm{~h}$. Cell proliferation was measured with a BrdU assay (Abcam UK): $\operatorname{BrdU}(10 \mu \mathrm{M})$ was added to each well and incubated for $12 \mathrm{~h}$, and the BrdU signal was calculated after absorbance detection at $450 \mathrm{~nm}$.

Cells were plated in 96-well plates and exposed to the indicated concentrations of PAC $(0.2,0.4,0.6 \mu \mathrm{M})$ or vehicle control (DMSO) for 12, 24, and $36 \mathrm{~h}$. Cell viability was examined with an MTS assay (Sigma, USA), $20 \mu \mathrm{L}$ of MTS was added to each well and incubated for $1 \mathrm{~h}$, after which the absorbance of the MTS signal was calculated after absorbance detection at $490 \mathrm{~nm}$.

\section{Colony Formation Assay}

Cells were treated with the indicated amount of PAC $(0.1,0.2$, $0.4,0.6 \mu \mathrm{M}$ ) or vehicle control (DMSO) for $24 \mathrm{~h}$ and cultured at a density of 500 cells/well in 6-well plates. The medium was changed every $3 \mathrm{~d}$. Two weeks later, cell colonies were stained with Giemsa Stain Solution (Solarbio, CN). Visible colonies were photographed and counted using a GelDocTMXR ${ }^{+}$Molecular Imager system (BioRad, USA).

\section{Hoechst 33258 Staining}

The morphology of apoptotic cells was observed using a Hoechst 33258 Staining Kit following the manufacturer's instructions and an IX73-AF12/PH fluorescence microscope (Olympus, JAN).

\section{Flow Cytometry}

Cells were incubated in 6-well plates and exposed to the indicated concentrations of PAC or vehicle control for $24 \mathrm{~h}$. Apoptosis and cell cycle analyses were performed using an Annexin-V FITC/PI Staining Kit and Cell-Cycle Detection Kit (KeyGENBiotech, CN) following the manufacturer's instructions. All data were evaluated by FlowJo software (BD Biosciences, USA).

\section{Real-Time RT-PCR Analysis}

Total RNA was extracted from cells with a Trizol Reagent Kit (Invitrogen) at the indicated time points, and Notch1, Notch2, and control $\beta$-actin complementary DNA syntheses were performed with a RevertAid First Strand cDNA Synthesis Kit (Fermentas, USA). RT-PCR was carried out as reported previously (15). The primer pairs used are listed in Supplementary Table 1.

\section{Western Blotting}

Samples were prepared for western blotting according to the protocol by Sun et al. (16). Primary antibodies against $\beta$ actin, Notch1, Notch2, N1ICD, N2ICD, HES1, PTEN, p-PTEN, AKT, p-AKT, Bax, Bcl-2, Caspase-3, and Caspase- 9 and the secondary antibody (goat anti-rabbit antibody conjugated to horseradish peroxidase) were all purchased from Cell Signaling (Massachusetts, USA).

\section{Plasmids and Cell Transfection}

Notch1-NTM (NM017617) and Notch2-NTM (VH879689) expression plasmids were constructed by Vigene Biosciences (Jinan, CN). The plasmids were transfected into cells using Lipofectamine 2000 following the manufacturer's recommended protocols.

\section{Observation of the Antitumor Effect in vivo}

Animal experiments were approved by the Animal Care and Use Committee of Hainan Medical College and abided by animal protocols (grant number: HYLL-2019-033). MGC-803 and SGC7901 tumor models were generated using male BALB/c-nude mice at 7 weeks of age. The mice were subcutaneously inoculated in the right inguinal region with the corresponding GC cells $(1 \times$ $\left.10^{6}\right)$. When the tumor was visible $\left(\sim 50 \mathrm{~mm}^{3}\right.$ at $\left.\sim 7 \mathrm{~d}\right)$, the mice 
were randomly divided into two groups (5 in each group) for treatment with $0.1 \mathrm{~mL}$ DMSO (PAC $0 \mathrm{mg} / \mathrm{kg}$ ) or PAC $(30 \mathrm{mg} / \mathrm{kg}$ ) via the tail vein once every $3 \mathrm{~d}$. The treatments were performed 7 times, and the mice were observed for 4 weeks. Tumor volumes were assessed every week using the following formula: tumor volume $=\left(\right.$ width $^{2} \times$ length $) / 2$.

\section{Statistical Analysis}

Statistical analysis was carried out with Prism 7. Differences were analyzed using one-way ANOVA or a two-sample equalvariance Student's $t$-test. Data are expressed as the mean $\pm S D$, and $P<0.05$ was deemed to be statistically significant.

\section{RESULTS}

\section{Penicilazaphilone C (PAC) Suppresses the Proliferation of GC Cells}

To explore the anticancer activities of PAC, we examined cell growth and proliferation in two GC cell lines, MGC-803 and SGC-7901. The cells were exposed to different amounts of PAC for the indicated durations, followed by analysis by MTT, BrdU, MTS, and colony formation assays to examine whether PAC affected cell viability and proliferation. MTT assays showed that viability in both MGC-803 and SGC-7901 was dose-dependently suppressed by PAC compared to that in controls (Figure 1B). Subsequently, similar inhibitory effects of PAC treatment for $24 \mathrm{~h}$ were observed based on BrdU assays (Figure 1C). Moreover, the results of MTS assays indicated that the cytotoxic effects of PAC on these two GC cells line not only increased with increasing dose but also with exposure time (Figure 1D). Additionally, microscopy images showed obvious cellular shrinkage after PAC treatment, with significantly decreased cellular attachment in comparison with controls (Figure 1F). Furthermore, colony formation assays suggested that PAC treatment markedly suppressed proliferation in MGC803 and SGC-7901 cells compared to controls (Figure 1E). We also assessed whether PAC is toxic to normal human gastric mucosal cells (GES-1), and the results showed that PAC at $<1.6 \mu \mathrm{M}$ was not cytotoxic to normal gastric cells; the dose that produced obvious cytotoxicity was $\sim 3.2 \mu \mathrm{M}$, more than eightfold the dose used in our assays (Supplementary Figure 1). These findings indicate that PAC specifically suppresses the viability and growth of GC cells. The $\mathrm{IC}_{50}$ of PAC was $\sim 0.4 \mu \mathrm{M}$ in both MGC-803 and SGC-7901, as shown in Figure 1B.

\section{PAC Induces Apoptosis in GC Cells}

Next, we explored whether inhibition of cell proliferation after PAC treatment was accompanied by apoptosis. We evaluated changes in nuclear morphology and the percentage of apoptotic MGC-803 and SGC-7901 cells by using a Hoechst 33258 staining solution. After PAC treatment, chromatin condensation and cell nuclear shrinkage, which are typical apoptotic morphological characteristics, were significantly increased in the two GC cell lines compared to the controls (Figure 2A). Similar, apoptotic characteristics in GC cells were observed based on annexin V/PI staining after PAC treatment (Figure 2B). To further confirm the apoptotic effects of PAC on MGC-803 and SGC-7901 cells, we performed flow cytometry after annexin V-FITC/PI staining and found that PAC caused both early and late apoptosis in a dosedependent manner. As shown in Figure 2C, the percentage of apoptotic MGC-803 cells was $1.07 \%$ in the control group and reached $26.78 \pm 1.21,38.15 \pm 1.36$, and $66.92 \pm 2.53 \%$ in the treatment groups; for SGC-7901 cells, the percentage of apoptotic cells was $2.08 \%$ in the control group and $14.07 \pm 0.75,18.13 \pm$ 1.07 , and $30.23 \pm 1.23 \%$ in the treatment groups. These results prove that the percentage of apoptotic cells increased markedly with increasing doses of PAC. Our findings indicate that PAC can induce apoptosis in GC cells.

\section{PAC Causes Cell-Cycle Arrest in G1 Phase}

To investigate whether the inhibition of cell proliferation due to PAC treatment was associated with cell cycle arrest, the influence of PAC on the cell cycle in MGC-803 and SGC-7901 cells was examined. Upon PAC treatment, G1-phase cells were obviously increased compared with those in the control group. As shown in Figure 2D, the percentage of MGC-803 cells in G1 and S phases was increased compared to those in the control group; however, only the percentage of SGC-7901 cells in G1 phase was increased. Our findings show that PAC arrested GC cells in the G1 phase of the cell cycle.

\section{Expression of Notch1 and Notch2 in GC Cell Lines}

To evaluate Notch signaling activation in GC cells, we examined relative expression of Notch1 and Notch2 at the mRNA and protein levels in five GC cell lines and GES-1 cells (Supplementary Figures 2a,b). Expression of both Notch1 and Notch 2 mRNA and protein was obvious in the five GC cell lines. Compared to GES-1 cells, Notch1 RNA and protein expression was higher in the various gastric cancer cells, whereas Notch2 expression was variable. For example, Notch2 expression was lower in MKN-28, MKN-45 and MGC-803 cells but higher in SGC-7901 and AGS compared to GES-1 cells, which was consistent with previous studies $(17,18)$, Therefore, we selected a cell lines with high Notch2 expression (SGC-7901) and a cell lines with low Notch2 expression (MGC-803) as the for subsequent experiments.

\section{PAC Induces Dephosphorylation of AKT and Blocks Notch Signaling in GC Cells}

Recent reports have revealed that the PTEN/AKT signaling pathway is involved in the regulation of apoptosis, and there are many reports that antitumor drugs induce apoptosis by inhibiting this pathway $(19,20)$. AKT is an effector molecule downstream of PTEN (21). Simultaneous phosphorylation of Thr308 and Ser473 is required for complete activation of AKT, and activated AKT can inhibits pro-apoptotic factors, such as Bax, Bad, and caspase- 9 , and activate anti-apoptotic factors, such as Bcl-2 and Bcl-xL (15). To examine whether PAC-induced apoptosis is stimulated by a PTEN/AKT-related pathway in GC cells, PTEN/AKT axis-related proteins were detected by western blotting. As shown in Figure 3A, AKT phosphorylation levels were significantly decreased upon PAC treatment in a dose-dependent manner compared to controls, though total 


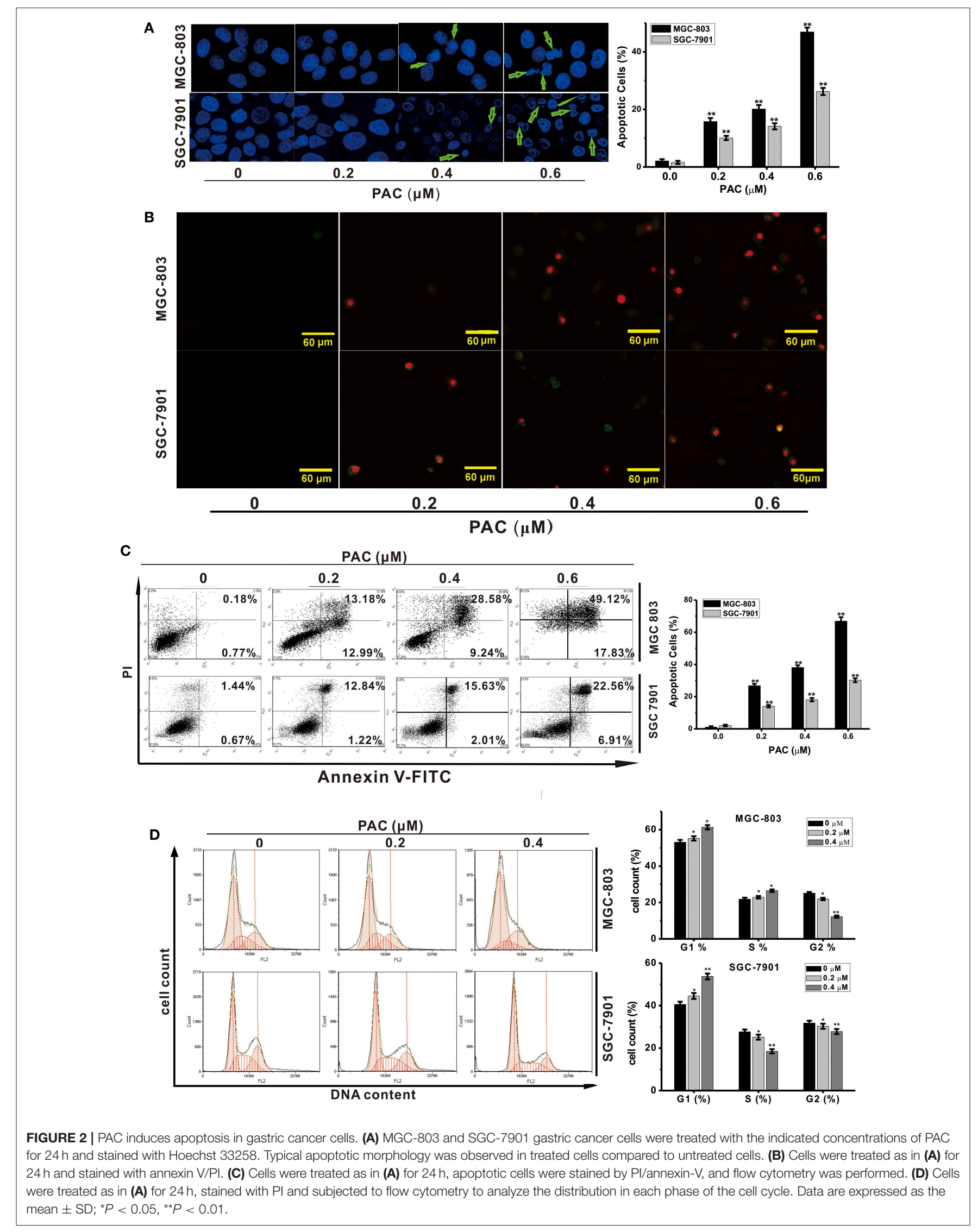



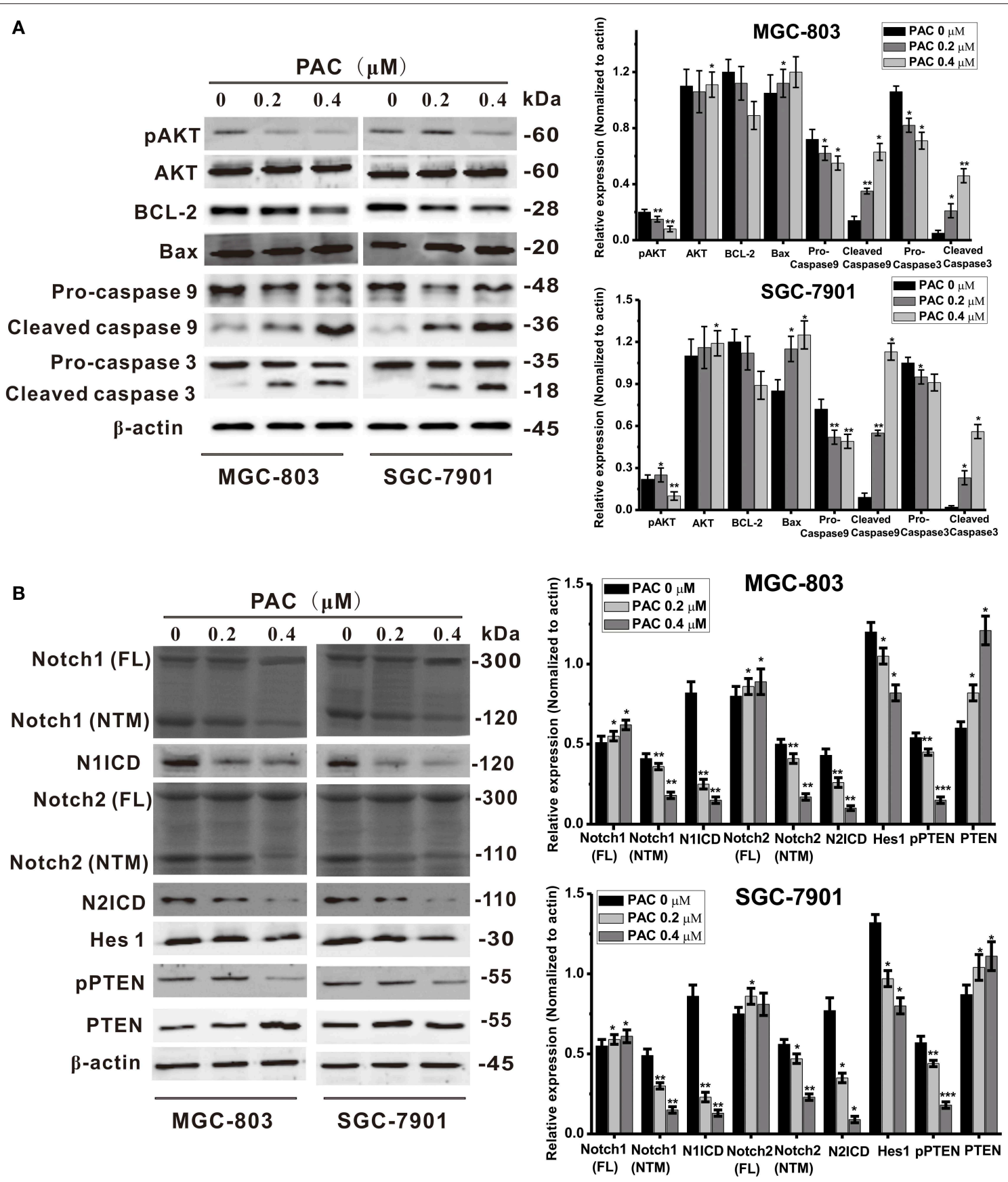

FIGURE 3 | PAC induces apoptosis through inhibition of the Notch/PTEN/AKT pathway. (A) Expression levels of apoptosis-related proteins were detected by western blot analysis after MGC-803 and SGC-7901 gastric cancer cells were treated with the indicated concentrations of PAC. (B) Expression levels of Notch signaling pathway-related proteins were detected by western blot analysis after MGC-803 and SGC-7901 gastric cancer cells were treated with the indicated concentrations of PAC. Data are expressed as the mean $\pm \mathrm{SD} ;{ }^{\star} P<0.05,{ }^{\star \star} P<0.01,{ }^{\star \star *} P<0.001$.

AKT expression was increased in a dose-dependent manner. In addition, apoptosis was observed after PAC treatment, which was confirmed by increased cleavage of caspase- 9 and -3 as well as the increased ratio of $\mathrm{Bax} / \mathrm{Bcl}-2$ at $24 \mathrm{~h}$ compared to controls.

Because Notch signaling impacts the PTEN/AKT signaling axis, we also assessed the expression of PTEN- and Notchrelated proteins. As shown in Figure 3B, compared to controls, the protein content of full-length Notch1 and Notch2 increased slightly in both GC cell lines, but the transmembrane fragments of Notch1 (Notch1-NTM) and Notch2 (Notch2-NTM) were notably decreased and dramatically decreased in N1ICD and N2ICD after $24 \mathrm{~h}$ of PAC treatment. Consistently, the expression levels of the target gene Hes1 were also significantly decreased in the PAC-treated group compared to those in the controls. 
A

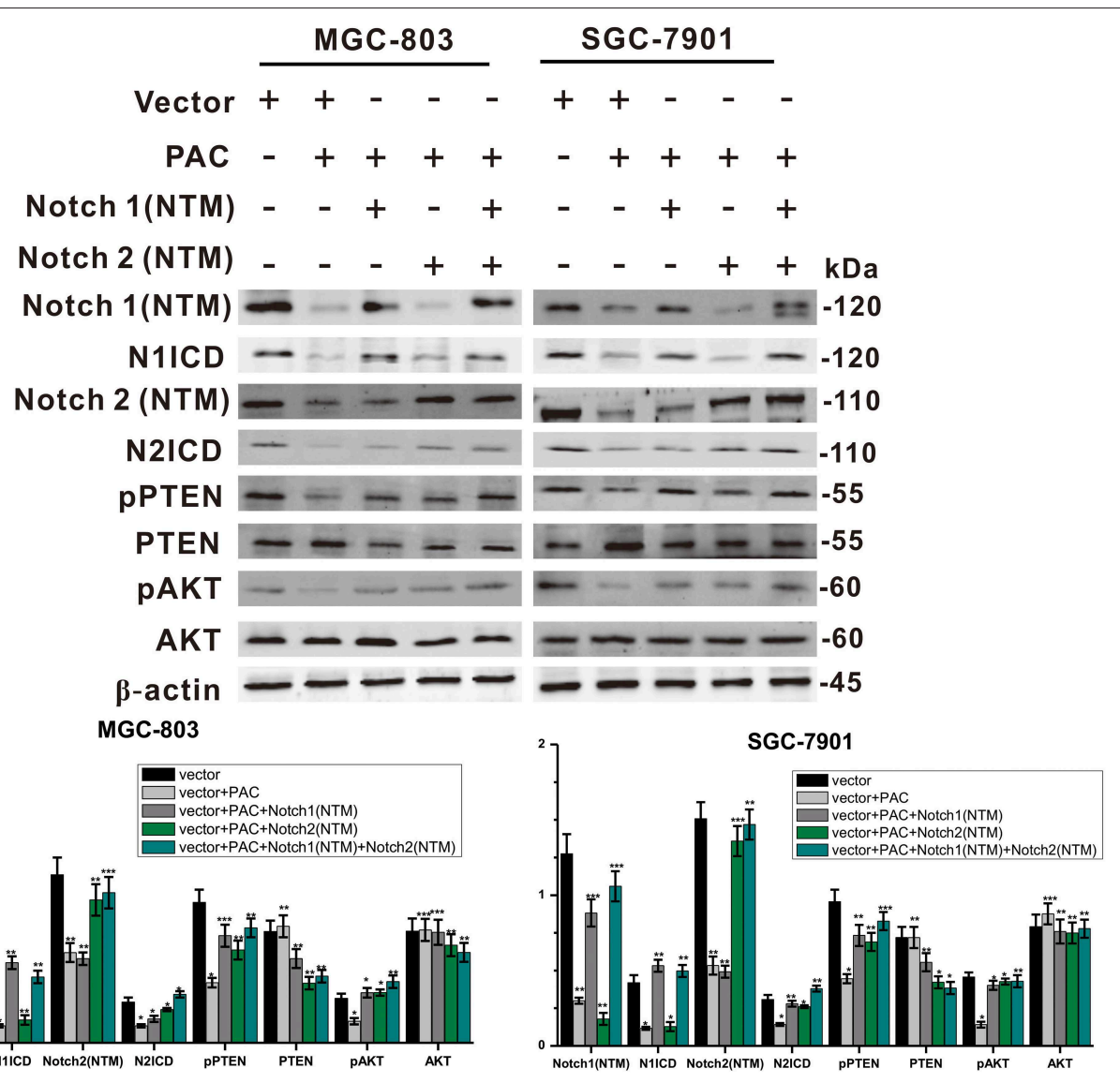

B

$\operatorname{PAC}(0.4 \mu \mathrm{M})$

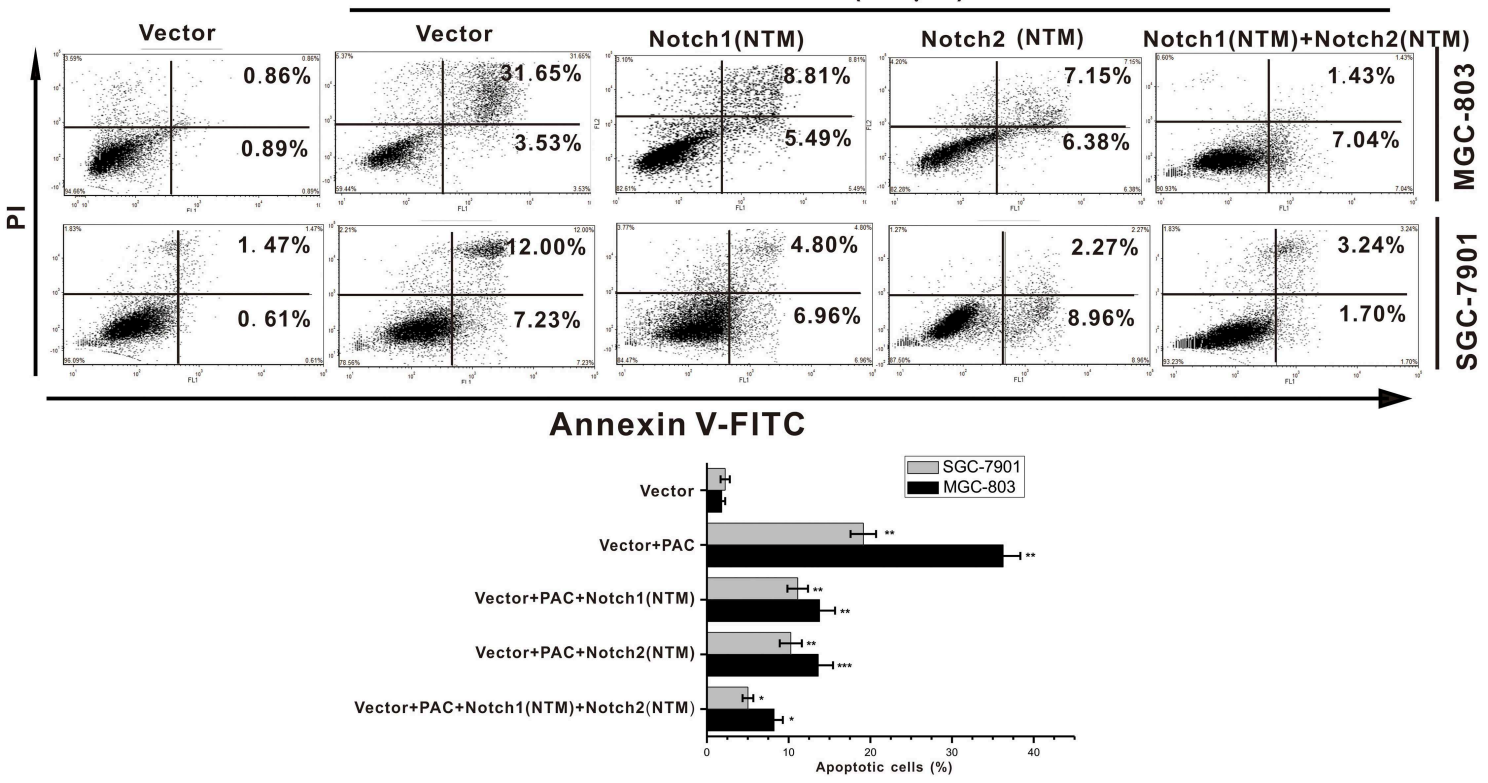

FIGURE 4 | PAC induces apoptosis in gastric cancer cells through blockage of the PTEN/AKT axis. (A) Cells were treated with or without PAC (0.4 $\mu$ M) in combination with transfection of the Notch1 (NTM) plasmid and/or Notch2 (NTM) plasmid for 24 h. Notch1 (NTM), N1ICD, Notch2 (NTM), N2ICD, Hes1, PTEN, phosphorylated PTEN (pPTEN), AKT, and phosphorylated AKT (PAKT) were detected by western blotting. (B) Cells were treated with or without PAC (0.4 $\mu \mathrm{M})$ in combination with transfection of the Notch1 (NTM) plasmid and/or Notch2 (NTM) plasmid for $24 \mathrm{~h}$, apoptotic cells were stained by PI/annexin-V, and flow cytometry was performed. Data are expressed as the mean $\pm \mathrm{SD}$; ${ }^{\star} P<0.05,{ }^{\star \star} P<0.01,{ }^{\star \star \star} P<0.001$. 
In contrast, as a negative regulator downstream of PTEN, PAC slightly increased PTEN protein expression, though PTEN phosphorylation was significantly decreased compared to the controls. We also examined changes in the levels of the Notch ligands Jagged 1, Jagged 2, and DLL4 and found that the protein expression of these ligands did not change significantly upon PAC treatment (Supplementary Figure 3). These findings suggest that PAC treatment may block the initial proteolytic events in Notch receptor activation.

\section{Overexpression of the Transmembrane Fragment of Notch Reverses the Effect of PAC on Apoptosis}

To examine the mechanism underlying the suppression of GC cell proliferation and the induction of apoptosis by PAC, GC cells were transfected with a Notch1 (NTM) and/or Notch2 (NTM) overexpression plasmid, with contrasting expression profiles for proteins in the Notch/PTEN/AKT axis. As presented in Figure 4A, overexpression of Notch1 (NTM) and/or Notch2 (NTM) resulted in a significant increase in NICD, Hes1, pPTEN, and pATK in both MGC-803 and SGC-7901 cells compared to controls. Conversely, protein expression of PTEN and AKT was slightly decreased in the overexpression groups compared to that in the controls. Moreover, compared to controls overexpression of Notch1 and/or Notch2 notably reduced PACinduced apoptosis in the two GC cell lines transfected with plasmids (Figure 4B). Our results strongly indicate that PAC induces apoptosis in GC cells by blocking the initial proteolytic cleavage of the Notch receptor and release of NICD into the cytoplasm.

\section{PAC for Tumor Treatment in vivo}

To examine the antitumor activities of PAC in vivo, MGC-803 and SGC-7901 cells were inoculated into BALB/c-nude mice. This experiment clearly demonstrated that PAC is an effective in vivo antitumor drug. As depicted in Figures 5A,B, PAC treatment significantly suppressed tumor growth compared to that in the control group. Figure $5 \mathrm{C}$ also shows that the tumors in the PAC-treated group, which were collected at $28 \mathrm{~d}$, were smaller and weighed less than did those in the control group.

\section{DISCUSSION}

Despite the significant contributions of tumor chemotherapy and resection to gastric carcinoma treatment, genetic variation, and drug resistance reduce the efficacy of these treatments (22). The natural products of marine fungi are rich in unique structural types (23), and PAC is a novel natural compound with a special azaphilone chemical structure isolated from the marine fungus $P$. sclerotiorum M-22 by our research group that exhibits anticancer activity in several cancer cell lines. Although PAC exhibits a marked anticancer effect, it is a new azaphilone structure, and the ways by which PAC regulates cancer programmes remains unknown. In this study, we attempted to elucidate the underlying mechanisms by which PAC affects GC cells.
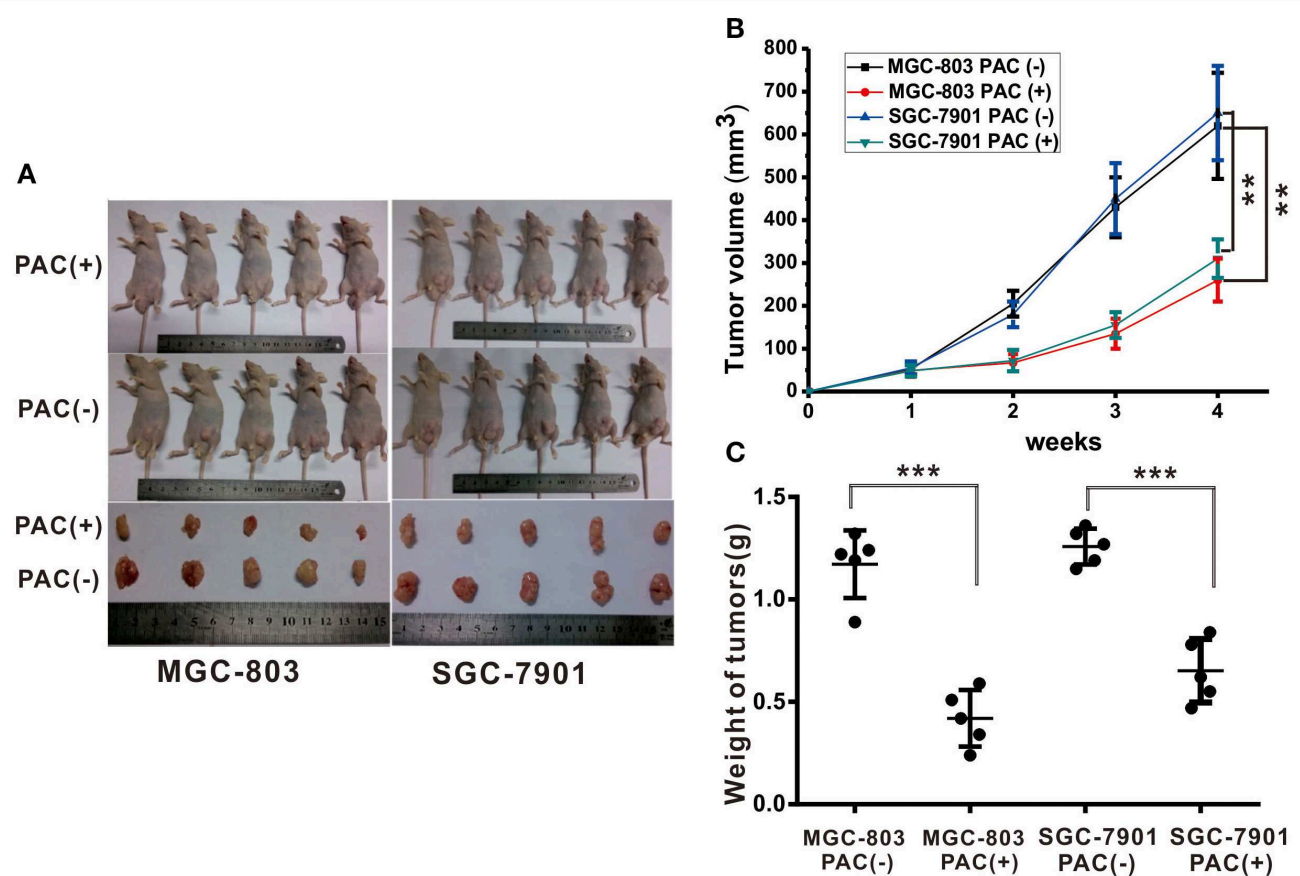

FIGURE 5 | PAC induced suppression of gastric tumor growth. Human MGC-803 and SGC-7901 gastric cancer cells were injected into the right flanks of Nu/Nu mice. When tumor volumes reached $50 \mathrm{~mm}^{3}$, the mice were treated with the indicated formulations every 3 days for 7 total treatments. (A) Pictures of mice taken after 4 weeks of treatment with the indicated therapies. (B) Tumor growth curves of all right and left flank tumors with the indicated treatments. (C) Tumor weight curves for the indicated therapies. Data are expressed as the mean $\pm \mathrm{SD} ;{ }^{\star \star} P<0.001,{ }^{\star \star \star} P<0.001$. 
As the most notable hallmark of tumor cells is uncontrolled growth (24), we first examined whether PAC is able to inhibit cancer cell growth (proliferation) by performing MTT, BrdU, MTS, and colony formation assays. The results showed that PAC obviously inhibited cell proliferation in MGC-803 and SGC-7901 cells in a dose- and time-dependent manner. Next, we found that PAC induces apoptosis and cell cycle arrest in the G1 phase by restraining AKT signaling, which is involved in cell proliferation and apoptosis. As expected, pAKT levels were decreased upon PAC treatment compared to that in controls. In contrast, the pro-apoptotic factors cleaved caspase -9 and -3 and the Bax/Bcl-2 ratio was increased in a dose-dependent manner after PAC treatment compared to controls. Notch signaling is predicted to be a target of PAC based on our endogenous tumor network model, and the regulation of Notch signaling plays a key role in tumor progression. Previous reports have shown that Notch signaling inhibits transcription of PTEN and activates the PI3K/AKT pathway $(21,25)$. Kim et al. (15) reported that the Notchtargeted protein Hes-1 suppresses PTEN expression through induced reversible phosphorylation of PTEN C-terminal sites and activates PI3K/AKT signaling in GC cells. Subsequently, we assessed the influence of PAC on Notch-related signaling. Upon PAC treatment, phosphorylation of PTEN and expression of Notch1 (NTM), Notch2 (NTM), N1ICD, N2ICD, and Hes1 were markedly decreased but the content of PTEN increased compared to controls. Additionally, we found that PAC treatments slightly enhanced the expression of the three ligands, full-length Notch1 and Notch2 compared to controls. These findings suggest that PAC blocks the first proteolytic events in Notch receptor activation. Furthermore, compared to controls, PAC-induced inhibition of Notch-related protein expression levels was promoted by overexpression of Notch1 (NTM) and/or Notch2 (NTM), though overexpression of Notch1 (NTM) and/or Notch2 (NTM) also suppresses the apoptosis caused by PAC. Our results strongly suggest that PAC treatment may inhibit the enzymatic cleavage that occurs during Notch receptor activation and then block NICD binding to target genes, ultimately exerting biological effects.

The binding of Notch receptors and ligands in adjacent cells triggers the Notch signaling pathway (26). As a result of binding, the receptor undergoes sequential proteolytic cleavages. First, the extracellular fragment is liberated from the extracellular surface by $\alpha$-secretase, and then the NICD is released

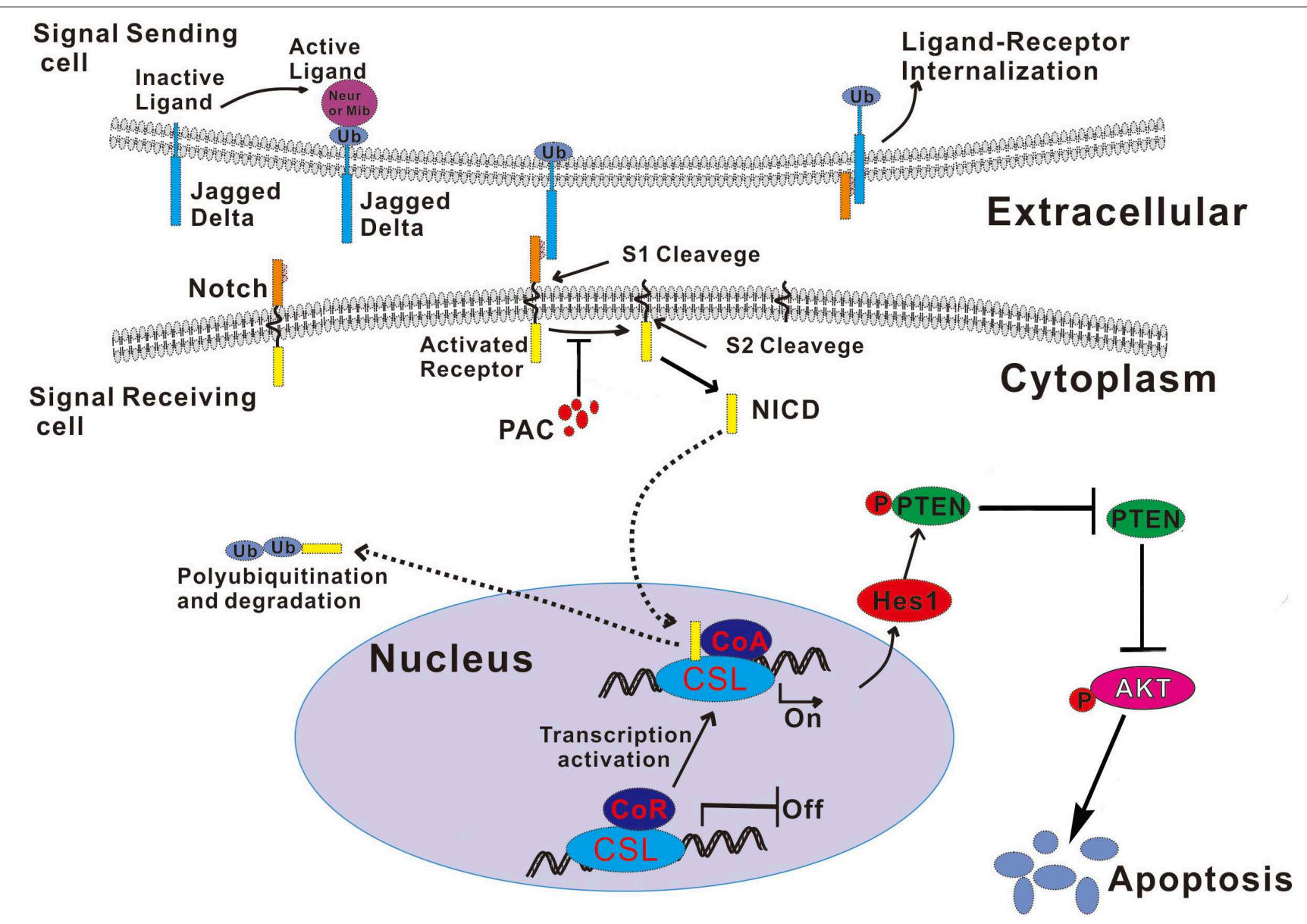

FIGURE 6 | Schematic illustration of the mechanism by which PAC induces apoptosis by blocked the Notch/PTEN/AKT axis in gastric cancer cells. Ub, ubiquitin. 
into the cytoplasm by $\gamma$-secretase (27); these two secretases belong to the ADAM (a disintegrin and metalloproteinase) endopeptidase family (28). The results of this experiment indicate that PAC can inhibit Notch receptor proteolytic activation by $\alpha$-secretase, subsequently blocking the Notch pathway. However, it remains unclear how PAC interacts with $\alpha$-secretase to inhibit the activation of $\alpha$-secretase. It is also not known whether PAC can inhibit other ADAM endopeptidases. Thus, the mechanisms underlying PAC-mediated $\alpha$-secretase activities are still poorly understood, and further research is needed.

In summary, this study demonstrates that PAC is a potential therapy for GC. PAC induces apoptosis by blocking the proteolytic cleavage of the activated Notch receptor and further influences the PTEN/AKT axis. Our experimental data reveal the molecular mechanism underlying the anticancer activity of PAC (summarized in Figure 6) and strongly suggest that PAC, as an inhibitor of Notch signaling, is a potential alternative drug for the treatment of GC patients.

\section{DATA AVAILABILITY STATEMENT}

All datasets generated for this study are included in the article/Supplementary Material.

\section{REFERENCES}

1. Siegel RL, Miller KD, Jemal A. Cancer statistics, 2019. CA Cancer J Clin. (2019) 69:7-34. doi: 10.3322/caac.21551

2. Chen W, Zheng R, Baade PD, Zhang S, Zeng H, Bray F, et al. Cancer statistics in China, 2015. CA Cancer J Clin. (2016) 66:115-32. doi: 10.3322/caac.21338

3. Zhang G, Li J, Li S, Wang Y. Exploring spatial trends and influencing factors for gastric cancer based on bayesian statistics: a case study of Shanxi, China. Int J Environ Res Public Health. (2018) 15:1824. doi: 10.3390/ijerph150 91824

4. Rugge M, Meggio A, Pravadelli C, Barbareschi M, Fassan M, Gentilini M, et al. Gastritis staging in the endoscopic follow-up for the secondary prevention of gastric cancer: a 5-year prospective study of 1755 patients. Gut. (2019) 68:11-7. doi: 10.1136/gutjnl-2017-314600

5. Haag GM, Czink E, Ahadova A, Schmidt T, Sisic L, Blank S, et al. Prognostic significance of microsatellite-instability in gastric and gastroesophageal junction cancer patients undergoing neoadjuvant chemotherapy. Int J Cancer. (2019) 144:1697-703. doi: 10.1002/ijc.32030

6. Zhou SL, Wang M, Zhao HG, Huang YH, Lin YY, Tan GH, et al. Penicilazaphilone $\mathrm{C}$, a new antineoplastic and antibacterial azaphilone from the Marine Fungus Penicillium sclerotiorum. Arch Pharm Res. (2016) 39:16217. doi: 10.1007/s12272-016-0828-3

7. Ren J, Ding SS, Zhu A, Cao F, Zhu HJ. Bioactive azaphilone derivatives from the fungus Talaromyces aculeatus. J Nat Prod. (2017) 80:2199-203. doi: 10.1021/acs.jnatprod.7b00032

8. Zhao DL, Shao CL, Zhang Q, Wang KL, Guan FF, Shi T, et al. Azaphilone and diphenyl ether derivatives from a gorgonian-derived strain of the fungus Penicillium pinophilum. J Nat Prod. (2015) 78:2310-4. doi: 10.1021/acs.jnatprod.5b00575

9. Bai J, Lu Y, Xu YM, Zhang W, Chen M, Lin M, et al. Diversityoriented combinatorial biosynthesis of hybrid polyketide scaffolds from azaphilone and benzenediol lactone biosynthons. Organ Lett. (2016) 18:12625. doi: 10.1021/acs.orglett.6b00110

10. Uenishi GI, Jung HS, Kumar A, Park MA, Hadland BK, McLeod E, et al. NOTCH signaling specifies arterial-type definitive hemogenic

\section{ETHICS STATEMENT}

Animal experiments and protocol followed the guidelines and regulations set by the Animal Care and Use Committee of Hainan Medical College (Grant number: HYLL-2019-033).

\section{AUTHOR CONTRIBUTIONS}

MW: data analysis and interpretation. HZ: data collection and analysis. YL and ZX: data collection. JH: manuscript writing and polishing. SZ: conception and design, data analysis and interpretation, and writing. All authors read and approved the final manuscript.

\section{FUNDING}

This work was supported by the National Natural Science Foundation of China (Grant No. 81560484) and the Key Research and Development Project of Hainan Province (Grant No. ZDYF2019177).

\section{SUPPLEMENTARY MATERIAL}

The Supplementary Material for this article can be found online at: https://www.frontiersin.org/articles/10.3389/fonc. 2020.00116/full\#supplementary-material

endothelium from human pluripotent stem cells. Nat Commun. (2018) 9:1828. doi: 10.1038/s41467-018-04134-7

11. Wang K, Zhang Q, Li D, Ching K, Zhang C, Zheng X, et al. PEST domain mutations in Notch receptors comprise an oncogenic driver segment in triplenegative breast cancer sensitive to a gamma-secretase inhibitor. Clin Cancer Res. (2015) 21:1487-96. doi: 10.1158/1078-0432.CCR-14-1348

12. Nwabo Kamdje AH, Mosna F, Bifari F, Lisi V, Bassi G, Malpeli G, et al. Notch3 and Notch-4 signaling rescue from apoptosis human B-ALL cells in contact with human bone marrow-derived mesenchymal stromal cells. Blood. (2011) 118:380-9. doi: 10.1182/blood-2010-12-326694

13. Shih Ie M, Wang TL. Notch signaling, gamma-secretase inhibitors, and cancer therapy. Cancer Res. (2007) 67:1879-82. doi: 10.1158/0008-5472.CAN-06-3958

14. Luca VC, Kim BC, Ge C, Kakuda S, Wu D, Roein-Peikar M, et al. NotchJagged complex structure implicates a catch bond in tuning ligand sensitivity. Science. (2017) 355:1320-4. doi: 10.1126/science.aaf9739

15. Kim SJ, Lee HW, Baek JH, Cho YH, Kang HG, Jeong JS, et al. Activation of nuclear PTEN by inhibition of Notch signaling induces G2/M cell cycle arrest in gastric cancer. Oncogene. (2016) 35:251-60. doi: 10.1038/onc.2015.80

16. Sun Y, Huang YH, Huang FY, Mei WL, Liu Q, Wang CC, et al. 3'-epi12beta-hydroxyfroside, a new cardenolide, induces cytoprotective autophagy via blocking the Hsp90/Akt/mTOR axis in lung cancer cells. Theranostics. (2018) 8:2044-60. doi: 10.7150/thno.23304

17. Xiao HJ, Ji Q, Yang L, Li RT, Zhang C, Hou JM. In vivo and in vitro effects of microRNA-124 on human gastric cancer by targeting JAG1 through the Notch signaling pathway. J Cell Biochem. (2018) 119:2520-34. doi: 10.1002/jcb.26413

18. Wu X, Liu W, Tang D, Xiao H, Wu Z, Chen C, et al. Prognostic values of four Notch receptor mRNA expression in gastric cancer. Sci Rep. (2016) 6:28044. doi: $10.1038 /$ srep28044

19. Palomero T, Dominguez M, Ferrando AA. The role of the PTEN/AKT Pathway in NOTCH1-induced leukemia. Cell Cycle. (2008) 7:965-70. doi: $10.4161 /$ cc.7.8.5753

20. Lee HW, Kim SJ, Choi IJ, Song J, Chun KH. Targeting Notch signaling by gamma-secretase inhibitor I enhances the cytotoxic effect of 5-FU in gastric cancer. Clin Exp Metast. (2015) 32:593-603. doi: 10.1007/s10585-015-9730-5 
21. Zhang X, Yang Y, Feng Z. Suppression of microRNA-495 alleviates high-glucose-induced retinal ganglion cell apoptosis by regulating Notch/PTEN/Akt signaling. Biomed Pharmacother. (2018) 106:923-9. doi: 10.1016/j.biopha.2018.07.018

22. Boku N, Ryu MH, Kato K, Chung HC, Minashi K, Lee KW, et al. Safety and efficacy of nivolumab in combination with S-1/capecitabine plus oxaliplatin in patients with previously untreated, unresectable, advanced, or recurrent gastric/gastroesophageal junction cancer: interim results of a randomized, phase II trial (ATTRACTION-4). Ann Oncol. (2019) 30:250-8. doi: 10.1093/annonc/mdy540

23. de la Calle F. Marine microbiome as source of natural products. Microb Biotechnol. (2017) 10:1293-6. doi: 10.1111/1751-7915.12882

24. Hanahan D, Weinberg RA. Hallmarks of cancer: the next generation. Cell. (2011) 144:646-74. doi: 10.1016/j.cell.2011.02.013

25. Vaish V, Sanyal SN. Role of Sulindac and Celecoxib in the regulation of angiogenesis during the early neoplasm of colon: exploring PI3-K/PTEN/Akt pathway to the canonical Wnt/beta-catenin signaling. Biomed Pharmacother. (2012) 66:354-67. doi: 10.1016/j.biopha.2012.01.004

26. Hughes DP, Kummar S, Lazar AJ. New, tolerable gamma-secretase inhibitor takes desmoid down a notch. Clin Cancer Res. (2015) 21:7-9. doi: 10.1158/1078-0432.CCR-14-1660
27. Pietri M, Dakowski C, Hannaoui S, Alleaume-Butaux A, HernandezRapp J, Ragagnin A, et al. PDK1 decreases TACE-mediated alphasecretase activity and promotes disease progression in prion and Alzheimer's diseases. Nat Med. (2013) 19:1124-31. doi: 10.1038/n m.3302

28. Seegar TCM, Killingsworth LB, Saha N, Meyer PA, Patra D, Zimmerman $\mathrm{B}$, et al. Structural basis for regulated proteolysis by the alphasecretase ADAM10. Cell. (2017) 171:1638-48.e7. doi: 10.1016/j.cell.2017. 11.014

Conflict of Interest: The authors declare that the research was conducted in the absence of any commercial or financial relationships that could be construed as a potential conflict of interest.

Copyright (c) 2020 Wang, Zhao, Hu, Xu, Lin and Zhou. This is an open-access article distributed under the terms of the Creative Commons Attribution License (CC BY). The use, distribution or reproduction in other forums is permitted, provided the original author(s) and the copyright owner(s) are credited and that the original publication in this journal is cited, in accordance with accepted academic practice. No use, distribution or reproduction is permitted which does not comply with these terms. 